\title{
Primat der ambulanten Behandlung der Borderline-Persönlichkeitsstörung
}

\author{
Primacy of Out-Patient Treatment of Borderline Personality Disorder
}

\section{Bibliografie}

Dol http://dx.doi.org/

10.1055/s-0029-1223521

Psychiat Prax 2010; 37:

108-110

(c) Georg Thieme Verlag KG

Stuttgart · New York .

ISSN 0303-4259

\section{Korrespondenzadressen}

Dipl.-Psych. Hans Gunia

Psychologischer Psycho-

therapeut

Heidelberger Landstr. 171

64297 Darmstadt

hans.gunia@t-online.de

\section{Prof. Dr. Martin Bohus}

Klinik für Psychosomatik und Psychotherapeutische Medizin, Zentralinstitut für Seelische Gesundheit

15

68159 Mannheim

martin.bohus@zi-mannheim.de

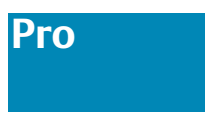

Die Behandlung von BorderlinePersönlichkeitsstörungen (BPS) gilt als sehr schwierig. Die Prävalenz der BPS wird in den USA mit 1-1,8\% angegeben. Auffallend ist eine hohe Suizidrate von 5$10 \%$ innerhalb von 15 Jahren und eine Selbstverletzungsrate von 69-80\% (zusammenfassend etwa Jerschke et al. [1]).

Jerschke et al. [1] fanden in ihrer Stichprobe häufige Therapieabbrüche $(47,4 \%)$ und Zwangseinweisungen (20,7\%). Aufgrund häufiger und langer Klinikaufenthalte schätzen Jerschke et al. die Kosten pro Jahr und Patient auf $12000 €$ (bezogen auf die letzten beiden Jahre vor Aufnahme auf einer speziellen DBT-Station an der Universitätsklinik Freiburg). Bei BPS verursacht eine relativ kleine Gruppe (1-1,5\%) relativ hohe Kosten durch Therapieabbrüche, häufige Klinikaufenthalte, disziplinarische Entlassungen, akute Behandlungsmaßnahmen usw.

Schon aus dieser Kurzübersicht ist ablesbar, dass Borderline-Patientinnen zu den sog. „Drehtürpatienten“ gehören und stationäre Settings häufig nutzen.

Die Nachteile stationärer Behandlungen sind meiner Ansicht nach dabei folgende:

1. Zu Beginn einer stationären Behandlung wird oft eine Klärung des Behandlungsziels vernachlässigt. Die Patienten werden ohne klar erkennbaren oder mit diffusem Auftrag behandelt. Das führt dazu, dass die Kriterien für eine Beendigung der Behandlung nicht operationalisiert sind und dass die Patienten nach der Krisenintervention weiter behandelt werden. Behandler haben dabei nicht selten Kognitionen wie: „Die Patientin bleibt im Krankenhaus, bis sie gesund ist." Abgesehen davon, dass dieser Anspruch uneinlösbar ist, tragen solche Kognitionen zur Hospitalisierung der Patientinnen bei.

2. Stationäre Settings sind dazu prädisponiert, dysfunktionales Verhalten im lerntheoreti- schen Sinn zu verstärken. Das heißt, Ärzte, Psychologen und Pflege reagieren auf selbstverletzendes Verhalten häufig mit zusätzlichen Gesprächsangeboten und verstärken so dysfunktionales Verhalten unabsichtlich. In einer Supervision reagierte ich deshalb ziemlich verblüfft, als ein Pfleger beiläufig fragte, ob es denn in Ordnung sei, dass sich eine Borderline-Patientin, eine Stunde nachdem sie sich geschnitten hatte, mit ihrem Notebook eine Pizza bestellte.

3. Borderline-Patientinnen konkurrieren in stationären Settings nicht selten um Zuwendung, indem sie versuchen, sich in der Quantität und Qualität ihrer Selbstverletzungen zu überbieten, was dann wiederum durch Zuwendung (s. o.) verstärkt wird.

4. Gelegentlich schauen sich Borderline-Patientinnen bei Mitpatientinnen „neues“ selbstverletzendes Verhalten ab.

5. Den Borderline-Patientinnen wird in stationären Settings häufig zu viel Verantwortung abgenommen. Es wird unterschätzt, dass diese Patienten schon eine längere Krankheitsgeschichte hinter sich haben und wahrscheinlich eine genauso lange noch vor sich, dass Patientinnen - obwohl ursächlich nicht „schuld an ihrer Misere“ - doch die Einzigen sind, die ihre Probleme lösen können.

6. Da die Borderline-Patientinnen aus ihrem Lebensumfeld herausgenommen werden, können gelernte neue Verhaltensweisen nicht oder nur ungenügend den Alltag generalisieren.

7. Es wird häufig vergessen, dass ein stationärer Aufenthalt auch eine Variante einer „Beziehung“ darstellt und dass Patientinnen kurz vor Abschluss der Behandlung deshalb nicht selten Angst „,vor dem Alleinsein“ haben und erneut dysfunktionales Verhalten zeigen. Ein optimaler Entlasszeitpunkt wird aus Angst oder Sorge der Behandler deshalb häufig verpasst und die 
Patientinnen werden länger im Krankenhaus belassen als es notwendig und sinnvoll wäre.

Auf der anderen Seite konnten wir in unserem ambulanten Darmstädter Netzwerk, das seit 1997 besteht und Borderline-Patientinnen mit der dialektisch behavioralen Therapie (DBT) behandelt, zeigen, dass man Borderline-Patientinnen sehr gut ambulant behandeln kann [2-4]. In einer eigenen Untersuchung [3] fanden wir innerhalb eines Jahres in einer Stichprobe von $33 \mathrm{~Pa}-$ tienten eine Einjahresabbrecherquote von nur $12 \%$. In der gleichen Stichprobe reduzierte sich die Anzahl der Patienten mit Suizidversuchen von 36 auf 6\%. Die Anzahl der Patienten, die sich selbst verletzten, reduzierte sich innerhalb eines Jahres von 79 auf $48 \%$. Die Anzahl der Patientinnen, die sich mindestens einmal wöchentlich verletzten, ging von 27 auf $4 \%$ zurück. Nach einem Jahr ambulante DBT zeigte sich ein durchschnittlicher Rückgang der Dauer der stationären Aufenthalte von 2,57 auf 0,35 Wochen. Bei einem angenommenen Tagessatz von $351 €$, überschlagen auf die 33 Patienten und gegengerechnet mit den Kosten, die für die ambulante Therapie mit DBT zu veranschlagen sind, ergibt sich pro Patient und Jahr eine durchschnittliche Ersparnis von 1566,42 € zugunsten der ambulanten Therapie.

Fairerweise muss man natürlich einräumen, dass es in der Bundesrepublik bislang leider nur einige wenige ambulante Netzwerke gibt, die sich die Behandlung von Patientinnen mit einer Borderline-Persönlichkeitsstörung zu ihrer Aufgabe machen und dass es demgegenüber viele auf BPS spezialisierte Stationen gibt, die diese Patientinnen mit der dialektisch behavioralen Therapie (DBT) behandeln. Für diese Spezialstationen gelten, da deren Teams sehr gut ausgebildet sind, die o.g. Nachteile in weit geringerem Ausmaß. Einschränkend muss man weiterhin sagen, dass es natürlich sinnvoll ist, für bestimmte Komorbiditäten spezialisierte stationäre Behandlungsangebote bereitzuhalten, bspw. BPS und schwere Abhängigkeitssymptome, BPS und schwere Essstörungen, BPS und Posttraumatische Belastungsstörungen, BPS und schwere dissoziative Symptome.

Die DBT als eine Therapie im Team mit sorgfältig aufeinanderabgestimmter Einzel- und Gruppentherapie passt hervorragend zu modernen Behandlungsstrukturen wie Integrierte Versorgung oder Medizinische Versorgungszentren und scheint deshalb das Mittel der Wahl als Methode für ambulante Borderline-Therapie zu sein. Unsere Erfahrungen zeigen, dass DBT im bundesrepublikanischen Versorgungssystem durchaus ambulant von Niedergelassenen angeboten werden kann. Die ambulante Versorgung ist möglich und ist gegenüber der stationären kostengünstiger. Der Gewinn für die teilnehmenden Therapeuten liegt darin, dass die Behandlung „schwieriger Patienten“ durch Entlastung im Team und durch strukturierte Behandlungskonzepte, im Vergleich zur Behandlung anderer Störungen, als nicht belastender erlebt wird [2]. Die Arbeit im Team und die Arbeit in und mit modernen Behandlungskonzepten bereichert die Arbeit ambulant arbeitender Psychotherapeuten. Der Gewinn für die Patientinnen liegt in der wohnortnahen Versorgung und darin, dass sie die neu erworbenen Fertigkeiten direkt vor Ort in der gewohnten Umgebung ausprobieren können. Der Gewinn für die Kostenträger liegt in einer kostengünstigen Versorgung, der ansonsten „sehr teuren“ Borderline-Patientinnen. Eine Hospitalisierung und Behandlungsabhängigkeit wird verhindert.

Es bleibt zu hoffen, dass sich in der Bundesrepublik Deutschland in den nächsten Jahren weitere ambulante Netze gründen und damit stationäre Behandlungen überflüssig machen.
Kontra

In der Bundesrepublik Deutschland werden derzeit jährlich etwa 4,5 Milliarden Euro für die stationäre Behandlung der Borderline-Störung (BPS) ausgegeben [5]. Vergegenwärtigt man sich diese Zahlen, und berücksichtigt man weiter, dass die Wahrscheinlichkeit einer Wiederaufnahme, nach einer unspezifischen stationären Behandlung bei etwa $80 \%$ liegt, so liegt die Vermutung nahe, dass unspezifische stationäre Behandlungen mehr schaden als nutzen. Und vor diesem Hintergrund ist die von Hans Gunia in diesem Heft vertretene Argumentationslinie, die klar gegen stationäre Behandlungen votiert, gut nachvollziehbar. Wenn wir dennoch der Bitte der Herausgeber nachkommen, Argumente für die stationäre Behandlung der BPS ins Feld zu führen, so sollte dies unter der dezidierten Annahme geschehen, dass die o.g. Daten, auf die sich auch Gunia bezieht, 1998 veröffentlicht wurden, also bevor es in Deutschland störungsspezifische Behandlungszentren gab, die sich an den Richtlinien der dialektisch behavioralen Therapie (DBT) orientierten.

Mittlerweile hat sich die Situation grundlegend geändert. Während 1998 knapp nur knapp die Hälfte der Psychiatrisch/Psychosomatischen Kliniken in Deutschland spezielle Angebote für BPS vorhielten, ist das mittlerweile bei über 3/4 der Kliniken der Fall [6]. Im Gegensatz zum 1998 noch berichteten überwiegenden Einsatz von tiefenpsychologischen Verfahren wird heute an erster Stelle DBT angewendet. Die Wirksamkeit der stationären DBT-Behandlungs-Programme ist mittlerweile sehr gut belegt. So konnte unsere Arbeitsgruppe in einer kontrollierten Studie in allen psychometrischen Variablen signifikante Unterschiede gegenüber der Warteliste zeigen, mit sehr großen Effektstärken (SCL-90-R GSI: d=0,83) [7]. Diese Daten konnten von unabhängigen Arbeitsgruppen repliziert werden [8] und zeigen sich auch im Langzeitverlauf stabil [9]. Der Deutsche Dachverband DBT (DDBT; www.dachverband-dbt.de) überprüft die Qualitätskriterien von stationären DBT-Behandlungseinheiten und vertritt relativ hohe Ansprüche an strukturelle, prozessurale und Ergebnisqualität: Dies ist in der deutschsprachigen Psychiatrie sicherlich einmalig. Kein anderes Behandlungsprogramm erhebt solch hohe Ansprüche. Und gerade die Tatsache, dass es derzeit in Deutschland trotz dieser hohen Qualitätsansprüche über 30 zertifizierte Behandlungseinheiten bzw. Behandlungsanwärter gibt, spricht dafür, dass die Klinikträger diese Bemühungen anerkennen und willens sind, die Qualität der Behandlung nachhaltig zu verbessern.

Aber auch die stationäre Behandlung für Borderline-Patienten nach den Kriterien der DBT erfordert spezifische Indikationsstellung:

1. Stationäre Krisenintervention

Ambulante Therapeuten, die mit Borderline-Patienten arbeiten, tun sich leichter, wenn sie mit Kliniken kooperieren, die kurzfristige stationäre Aufenthalte für die Krisenintervention bei suizidalen Krisen vorhalten. Diese stationären Aufenthalte sollten geplant und vom niedergelassenen Therapeuten initiiert sein. Dabei sollten diese sich auf die wenigen Situationen reduzieren, in welchen die Problemlösekompetenz den Patienten tatsächlich überfordern, sodass Suizid oder schwerwiegende Folgen drohen. Dies ist unter adäquaten therapeutischen Bedingungen äußerst selten der Fall. Die stationäre Krisenintervention sollte in der Regel nicht länger als eine Woche dauern. Der Fokus der Behandlung liegt auf der Problem- und Bedingungsanalyse der Krisensituation sowie der Erarbeitung von rasch wirksamen Bewältigungsstrategien. Dies erfordert enge Kooperation und Absprache mit dem niedergelassenen Kollegen. Es ist sinnvoll, die Station, 
auf der die Krisenintervention stattfindet, räumlich von einer psychotherapeutischen Station zu trennen. Keinesfalls sollte diese Krisenintervention als „Hintertüre“ für eine ungeplante stationäre Langzeittherapie missbraucht werden.

2. Stationäre oder teilstationäre Standard-DBT-Behandlungsprogramme

Wir können davon ausgehen, dass die ambulante Standard DBT (Einzeltherapie plus Skillsgruppe plus Supervision, plus Telefonberatung) ähnlich gute Wirkung entfaltet, wie stationäre Behandlungsprogramme. Nur leider sind derartige „DBT-Netzwerke“ in Deutschland nur in wenigen Städten etabliert (z.B. Darmstadt, Berlin, Köln). Rein statistisch kommt nur eine von etwa 1000 Borderline-Patientinnen in Deutschland in den Genuss einer ambulanten störungsspezifischen Behandlung. Vor diesem Hintergrund erscheint es tatsächlich sinnvoll, stationäre Behandlungsprogramme vorzuhalten, die innert 3 Monaten die wichtigsten Problembereiche von BPS-Patienten bearbeiten. Dies betrifft in aller Regel die Vermittlung von Fertigkeiten zur Bewältigung von Suizidalität, schweren Selbstverletzungen, Hochrisikoverhalten, aggressiven Durchbrüchen, sowie die Vermittlung von basalen sozialen Kompetenzen.

\section{Stationäre oder teilstationäre Spezial-DBT-Behandlungspro-} gramme

Auch wenn die ambulante oder stationäre Standard-DBT im Gruppenmittel gute Erfolge aufweist, so gibt es doch eine Reihe von Problemen, die eine zusätzliche Behandlung in einem Subgruppen-spezialisierten DBT Behandlungsprogramm erfordert.

Dies ist zum einen komorbide Posttraumatische Belastungsstörung nach langwierigem sexuellen Missbrauch in der Kindheit, unter welcher etwa 60\% der weiblichen BPD-Patienten in Deutschland leiden. Ohne spezifische Behandlung persistiert diese Problematik über Jahre, mit erheblichen Auswirkungen auf die Lebensqualität der Betroffenen. Am ZI Mannheim wurde in den letzten Jahren ein hoch spezialisiertes Behandlungsprogramm für diese Gruppe von Patientinnen entwickelt, das sehr gute Effektstärken (PDS ca. d =1,4) und gute Remissionsraten zeigt [10]. Derzeit wird dieses Programm an mehreren Kliniken in Deutschland etabliert, um diese Daten zu replizieren. Dennoch kann man schon jetzt konstatieren, dass Borderline-Patientinnen mit therapierefraktärer komorbider PTBS den Behandlungsversuch auf einer entsprechenden Spezialstation wagen sollten.

Zum zweiten erfordern schwerwiegende komorbide Essstörungen oft die Behandlung auf einer entsprechend ausgerichteten Station, welche beiden Problembereichen (Essstörung plus Störung der Affektregulation) gerecht wird. Dies trifft insbesondere den Problembereich der Anorexie, da es sich im klinischen Alltag oft zeigt, dass gerade mit der Gewichtszunahme die Störung der Emotionsregulation in den Vordergrund tritt. Wenn diese nicht zeitgleich fokussiert wird, kommt es gehäuft zu suizidalen Krisen oder schwerwiegenden Selbstverletzungen. An der Psychiatrischen Klinik in Lübeck wurde ein derartiges Konzept entwickelt und evaluiert [11].

Und schließlich erfordert das Problem der komorbiden Substanzabhängigkeit (26-84\%) Expertenwissen und spezifische Behandlung: Eine Untersuchung zur Effektivität von Standard-DBT bei Patienten mit BPS und komorbider substanzbezogener Störung reproduzierte die überlegene Wirksamkeit der DBT auf die Borderline-spezifische Symptomatik, zeigte jedoch keinen Einfluss auf Frequenz und Menge des Substanzkonsums [12]. Erst die Integration psychotherapeutisch wirksamer Methoden aus der Suchttherapie sowie die Ergänzung der DBT-Zielhierarchisierung um Ziele aus dem Suchtbereich, Erweiterung des Skillstrainings auf den Umgang mit Craving, die Einführung des Prinzips der Dialektischen Abstinenz sowie Attachmentstrategien, führte in 2 randomisierten Studien im ambulanten Setting zu einer signifikanten Verbesserung der klinischen Symptome aus beiden Störungsbereichen [13,14]. Kienast et al. [15] etablierten ein stationäre DBT-S-Programm. Ergebnisse zeigen hier ebenfalls eine Reduktion der Borderline-spezifischen Symptomatik sowie substanzabhängiger Verhaltensweisen bei schwer kranken Patienten. Zentrum der DBT-S in Deutschland ist die Klinik für Psychiatrie und Psychotherapie Hamburg Eilbek.

\section{Literatur}

1 Jerschke S, Meixner $K$, Richter $H$ et al. Zur Behandlungsgeschichte und Versorgungssituation von Patientinnen mit Borderline-Persönlichkeitsstörung in der Bundesrepublik Deutschland. Fortschr Neurol Psychiatr 1998; 12: 219-229

2 Gunia H, Huppertz M, Friedrich J et al. Dialektich Behaviorale Therapie von Borderline-Persönlichkeitsstörungen in einem ambulanten Netzwerk. Verhaltenstherapie und Psychosoziale Praxis 2000; 32: 651-662

3 Gunia H, Friedrich J, Huppertz M. Evaluation eines ambulanten DBTNetzwerks - Erste Ergebnisse. In: Merod R, Hrsg. Behandlung von Persönlichkeitsstörungen - Integration. Tübingen: dgvt-Verlag, 2005: 523-547

4 Friedrich J, Guina H, Huppertz M. Evaluation eines ambulanten Netzwerks für Dialektisch Behaviorale Therapie. Verhaltenstherapie und Verhaltensmedizin 2003; 24: 289-306

5 Bohus M. Zur Versorgungssituation von Borderline-Patienten in Deutschland. Persönlichkeitsstörungen Theorie und Therapie 2007; 11: $149-153$

6 Jacob GA, Allemann R, Schornstein $K$ et al. Zum gegenwärtigen Stand der stationären Behandlung von Borderline-Patienten in Deutschland. Psychiat Prax 2009; 36: 387-389

7 Bohus M, Haaf B, Simms T et al. Effectiveness of inpatient dialectical behavioral therapy for borderline personality disorder: a controlled trial. Behavior Research and Therapy 2004; 42: 487-499

8 Kröger C, Schweiger U, Sipos Vet al. Effectiveness of dialectical behaviour therapy for borderline personality disorder in an inpatient setting. Behaviour Research and Therapy 2006; 44: 1211-1217

9 Kleindienst N, Limberger MF, Schmahl C et al. Do improvements after inpatient dialectial behavioral therapy persist in the long term? A naturalistic follow-up in patients with borderline personality disorder. Journal of Nervous and Mental Diseases 2008; 196: 847-851

10 Dyer A, Priebe K, Steil R et al. Dialektisch Behaviorale Therapie zur Behandlung der Posttraumatischen Belastungsstörung mit schweren Störungen der Emotionsregulation. Verhaltentherapie und Psychosoziale Praxis 2009; 41: 283-307

11 Sipos $V$, Schweiger $U$. Dialektisch Behaviorale Therapie für Patienten mit Essstörung. Stuttgart: Kohlhammer, 2010

12 Van den Bosch LM, Verheul R, Schippers GM et al. Dialectical Behavior Therapy of borderline patients with and without substance use problems. Implementation and long-term effects. Addictive Behaviors 2002; 27: 911-923

13 Linehan MM, Schmidt H, Dimeff LA et al. Dialectical behavior therapy for patients with borderline personality disorder and drug-dependence. American Journal on Addictions 1999; 8: 279-292

14 Linehan MM, Dimeff LA, Reynolds SK et al. Dialectical behavior therapy versus comprehensive validation therapy plus 12-step for the treatment of opioid dependent women meeting criteria for borderline personality disorder. Drug and Alcohol Dependence 2002; 67: 13-26

15 Kienast T, Foerster J. Psychotherapy of personality disorders and concomitant substance dependence. Curr Opin Psychiatry 2008; 21: 619624 